\title{
Correction to: Technical and Industrial Applications of $\mathrm{CO}_{2}$
}

\author{
Jan Vansant and Peter-Wilhem Koziel
}

\section{Correction to: \\ Chapter 3 in: M. Aresta et al. (eds.), An Economy Based on Carbon Dioxide and Water, https://doi.org/10.1007/978-3-030-15868-2_3}

The original version of this chapter included figures without numbering and figure captions. Some of the figures needed to be replaced with higher resolution images due to poor readability. The correction now includes figure captions and numbering as well as high resolution figures where needed. In addition, typographical errors have been corrected. 